\title{
C-reactive protein as a potential biomarker for disease progression in dengue: a multi- country observational study
}

Nguyen Lam Vuong ${ }^{1,2}$, Huynh Thi Le Duyen ${ }^{1}$, Phung Khanh Lam, Dong Thi Hoai Tam', Nguyen Van Vinh Chau ${ }^{3}$, Nguyen Van Kinh" ${ }^{4}$, Ngoun Chanpheaktra ${ }^{5}$, Lucy Chai See Lum ${ }^{6}$, Ernesto Pleités ${ }^{7}$, Nick Keith Jones ${ }^{8}$, Cameron Paul Simmons ${ }^{1,9}$, Kerstin Rosenberger ${ }^{10}$, Thomas Jaenisch ${ }^{10}$, Christine Halleux ${ }^{11}$, Piero Luigi Olliaro ${ }^{12}$, Bridget Wills ${ }^{1,12}$ and Sophie Yacoub ${ }^{1,12^{*}}$ (D)

\begin{abstract}
Background: Dengue infection can cause a wide spectrum of clinical outcomes. The severe clinical manifestations occur sufficiently late in the disease course, during day 4-6 of illness, to allow a window of opportunity for risk stratification. Markers of inflammation may be useful biomarkers. We investigated the value of C-reactive protein (CRP) measured early on illness days 1-3 to predict dengue disease outcome and the difference in CRP levels between dengue and other febrile illnesses (OFI).

Method: We performed a nested case-control study using the clinical data and samples collected from the IDAMSconsortium multi-country study. This was a prospective multi-center observational study that enrolled almost 8000 participants presenting with a dengue-like illness to outpatient facilities in 8 countries across Asia and Latin America. Predefined severity definitions of severe and intermediate dengue were used as the primary outcomes. A total of 281 cases with severe/intermediate dengue were compared to 836 uncomplicated dengue patients as controls (ratio 1:3), and also 394 patients with OFI.

Results: In patients with confirmed dengue, median (interquartile range) of CRP level within the first 3 days was $30.2 \mathrm{mg} / \mathrm{L}$ (12.4-61.2 mg/L) (uncomplicated dengue, 28.6 (10.5-58.9); severe or intermediate dengue, 34.0 (17.471.8)). Higher CRP levels in the first 3 days of illness were associated with a higher risk of severe or intermediate outcome (OR 1.17, 95\% Cl 1.07-1.29), especially in children. Higher CRP levels, exceeding $30 \mathrm{mg} / \mathrm{L}$, also associated with hospitalization (OR 1.37, 95\% Cl 1.14-1.64) and longer fever clearance time (HR 0.84, 95\% Cl 0.76-0.93), especially in adults. CRP levels in patients with dengue were higher than patients with potential viral infection but lower than patients with potential bacterial infection, resulting in a quadratic association between dengue diagnosis and CRP, with levels of approximately $30 \mathrm{mg} / \mathrm{L}$ associated with the highest risk of having dengue. CRP had a positive correlation with total white cell count and neutrophils and negative correlation with lymphocytes, but did not correlate with liver transaminases, albumin, or platelet nadir.
\end{abstract}

Conclusions: In summary, CRP measured in the first 3 days of illness could be a useful biomarker for early dengue risk prediction and may assist differentiating dengue from other febrile illnesses.

Keywords: Dengue, Biomarker, C-reactive protein, Prognosis, Other febrile illness

\footnotetext{
* Correspondence: syacoub@oucru.org

'Oxford University Clinical Research Unit, Wellcome Trust Asia Programme,

Ho Chi Minh City, Vietnam

${ }^{12}$ Centre for Tropical Medicine and Global Health, Nuffield Department of

Medicine, University of Oxford, Oxford, UK

Full list of author information is available at the end of the article
}

(c) The Author(s). 2020 Open Access This article is distributed under the terms of the Creative Commons Attribution 4.0 International License (http://creativecommons.org/licenses/by/4.0/), which permits unrestricted use, distribution, and reproduction in any medium, provided you give appropriate credit to the original author(s) and the source, provide a link to the Creative Commons license, and indicate if changes were made. The Creative Commons Public Domain Dedication waiver (http://creativecommons.org/publicdomain/zero/1.0/) applies to the data made available in this article, unless otherwise stated. 


\section{Background}

Dengue, caused by one of the four dengue virus serotypes (DENV1-4), is globally the most important arboviral infection, in terms of geographic spread and number of infections [1]. An estimated 390 million infections now occur annually in over 100 countries, of which 96 million manifest as symptomatic dengue cases [2]. The clinical phenotype can vary from a relatively mild self-limiting febrile illness, to severe and occasionally life-threatening symptoms of bleeding, organ impairment, and vascular leakage leading to shock [3]. These severe manifestations occur sufficiently late in the course of the disease around defervescence, which occurs usually on day 4-6 following illness onset, to allow a potential window of opportunity to identify patients who may progress.

In areas of dengue transmission, yearly seasonal epidemics occur and can very quickly overwhelm health facilities, with potentially thousands of patients being reviewed daily. As the vast majority of symptomatic infections will result in a benign disease course, the ability to identify patients at high risk of progression, who are likely to benefit from early intervention with supportive therapy, has become the focus of intense research efforts in recent years.

Several small studies have attempted to identify biomarkers for dengue that will be cost-effective in resource-limited settings [4]. Recent evidence suggests that markers of inflammation may be useful as biomarkers. Studies have shown higher levels of C-reactive protein (CRP) in severe dengue versus non-severe dengue, with a CRP cutoff level of $30.1 \mathrm{mg} / \mathrm{L}$ (AUC, 0.938; $100 \%$ sensitivity, $76.3 \%$ specificity) [5]. In adult patients in Indonesia on the third day of fever, CRP was higher in those who developed plasma leakage, 10.1 (IQR 4.3$36.5)$ vs. 6.3 (IQR 3.0-21.6) $\mathrm{mg} / \mathrm{L}(p=0.014)$ [6]. Other studies using highly sensitive (hs) CRP did not find a difference between the severity grades [7]. Higher levels of CRP have also been found in patients with dengue compared to other viral illnesses [8].

A lack of harmonization between these studies has made the results difficult to compare, with varying assay techniques, viral serotypes, age of the participants, immune status, and illness day at the time of sampling. To provide a definitive answer as to the utility of CRP measurement for diagnosis and risk prediction in dengue, these results require validation in a large sample set including early and dynamic sampling, and using a standardized assay.

We hypothesized that (1) dengue patients with higher CRP levels in the early febrile phase are at higher risk of developing severe disease and (2) dengue patients have higher CRP levels than patients with other viral febrile illnesses.

\section{Methods}

Study design

A nested case-control study was performed using the clinical data and blood samples already collected from patients recruited to the observational study entitled "Clinical evaluation of dengue and identification of risk factors for severe disease" (IDAMS study, NCT01550016), for which the protocol has been published [9]. Briefly, this prospective multi-center observational study enrolled 7428 participants aged 5 years or more, presenting with a febrile illness consistent with dengue to outpatient health facilities in 8 countries across Asia and Latin America. Patients at the participating sites were eligible for enrollment if they met the following criteria: fever or history of fever for less than $72 \mathrm{~h}$ and clinical symptoms consistent with possible dengue, and had no localizing features suggesting an alternative diagnosis, e.g., pneumonia. Participants were then followed daily until resolution of their acute illness, with a standard schedule of blood samples obtained during the illness course. Hospital admission and individual case management were determined according to clinical need, with all interventions documented in the case report forms. Subsequently, each participant was assigned an overall severity grading using all available information; the system used is described in detail in Additional file 1 and is in line with the recent recommendation to use standardized endpoints for severe and intermediate dengue to ensure reproducibility and comparability of research findings [10].

\section{Study population}

Samples from participants enrolled at five study sites were selected, including Hospital for Tropical Diseases (HTD) (Ho Chi Minh City, Vietnam), National Hospital for Tropical Diseases (NHTD) (Hanoi, Vietnam), Angkor Hospital for Children (Siem Reap, Cambodia), University of Malaya Medical Centre (Kuala Lumpur, Malaysia), and Hospital Nacional de Niños Benjamin Bloom (El Salvador). Stored samples from patients who progressed to severe or intermediate dengue severity were selected as well as a comparison group of uncomplicated dengue and other febrile illnesses (OFI). This cohort included 38 severe and 243 intermediate severity cases (combined primary outcome of 281 cases). The 281 cases were compared to 839 uncomplicated dengue patients as controls (1:3), based on similar geographic and demographics and day of illness (DOI) making a total sample size of 1120 dengue cases. Confirmed dengue cases were compared to 400 patients with OFI, again from the same locations and with the same demographics and DOI. The study flowchart is shown in Fig. 1.

\section{Case definitions}

Dengue diagnostic criteria have been described elsewhere; briefly, laboratory-confirmed dengue was defined by either 


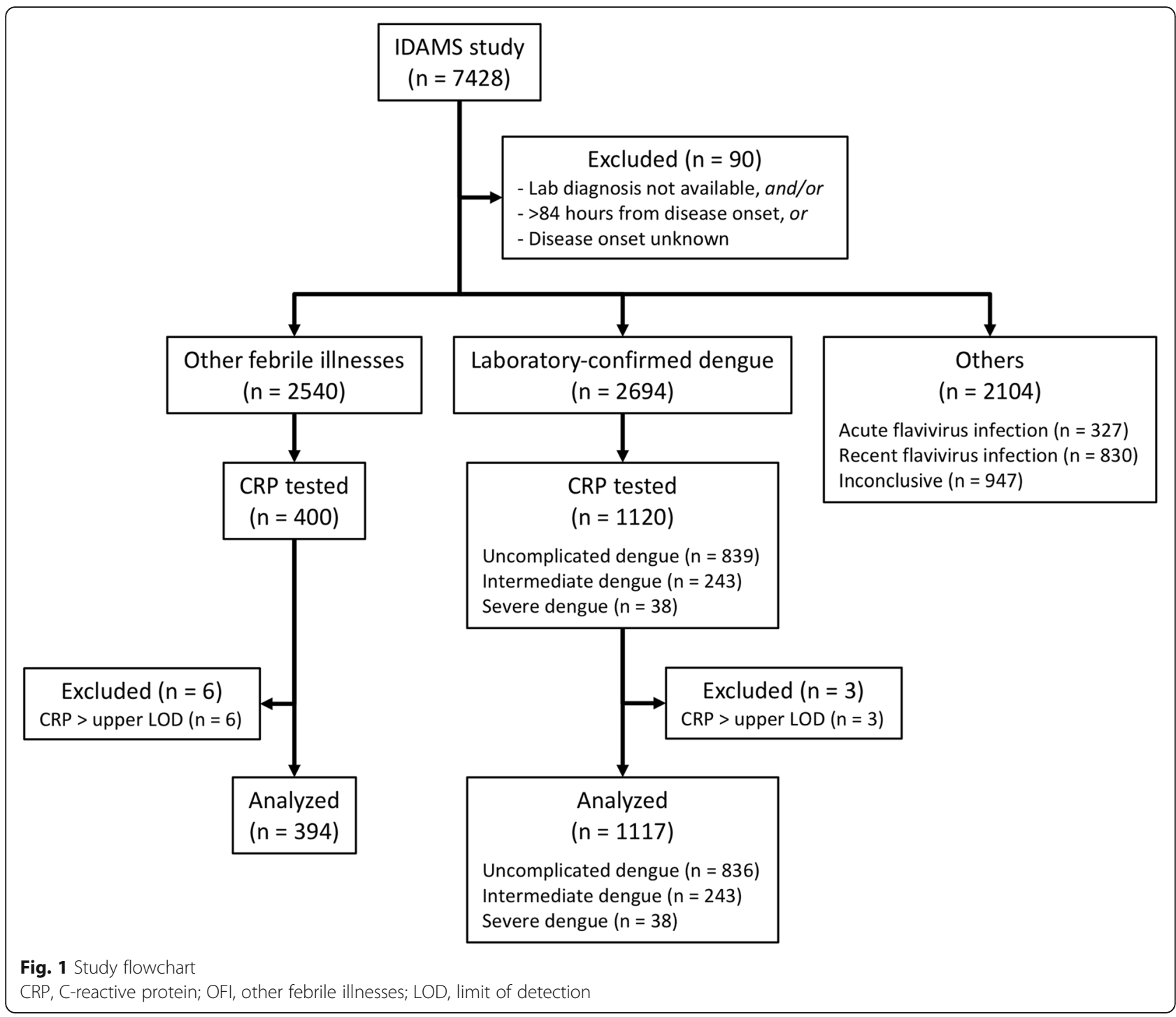

a positive RT-PCR assay or a NS1 ELISA (Platelia NS1, Biorad) test [9]. Patients with no laboratory evidence of acute or recent dengue were assigned as OFI. For all dengue cases, immune status was classified based on capture IgG results. A probable primary infection was defined by negative IgG results on two consecutive specimens on the acute and early phases of dengue infection, with at least one specimen being obtained during the second week since the onset of symptom. A probable secondary infection was defined by any positive IgG result from acute or early samples $[3,9]$. All other cases with the absence of suitable specimens at the appropriate time points were classified as inconclusive serology.

\section{Laboratory evaluation}

A full blood count was performed daily, while biochemistry tests were only performed at enrollment and then subsequently if clinically indicated. A research sample of
EDTA plasma was stored every other day. All research samples were processed at the different sites within $1 \mathrm{~h}$ of collection, centrifuged at $500 \mathrm{~g} / \mathrm{min}$ for $10 \mathrm{~min}$, and then stored at $-20^{\circ} \mathrm{C}$. All the samples were transferred on dry ice to OUCRU laboratory. CRP was measured on these stored samples at two time points: enrollment sample (illness day 1-3) and follow-up (day 10-21 postsymptom onset) using the same commercial assay according to the manufacturer's specifications (magnetic bead panel, cat. no. HCVD3MAG-67 K, Merck, Millipore, UK) on a Luminex 200 analyzer.

\section{Study endpoints}

To compare CRP levels between dengue and OFI groups, the outcome was laboratory-confirmed dengue or not. To investigate the association between CRP levels and severity in dengue patients, the primary outcome was severe or intermediate dengue, which was defined as any of severe 
or intermediate plasma leakage, bleeding, neurology involvement, liver involvement, or other major organ failure; all definitions were in accordance with current standard definition for use in dengue interventional trials [10] (see Additional file 1). Severe dengue events were rare, and the combined endpoint of severe or intermediate dengue is relevant for clinical practice, since less severe manifestations that require medical intervention contribute disproportionately to disease burden. The decision to hospitalize is very subjective, so we included this as a secondary outcome. Other secondary outcomes included severe dengue or dengue with warning signs according to WHO 2009, and fever clearance time. Fever clearance time was defined as the number of days from symptom onset to the day of defervescence (for patients still febrile at hospital discharge, fever clearance time was censored at the day of discharge).

\section{Statistical analysis}

Plasma CRP levels were transformed to base-2 logarithm $(\log 2)$ before analysis. Cox regression model was used to investigate fever clearance time while logistic regression model was used for other outcomes. The model comparing CRP levels between dengue and OFI groups was adjusted for age and day of illness (DOI) at enrollment. Other models for association between CRP levels and dengue severity were adjusted for age, DOI at enrollment, plasma viremia levels at enrollment, and immune status. Other factors, such as fluid infusion, bacteria coinfection, antibiotic usage, and comorbidity, had unclear potential relationship with either CRP level and clinical outcome, and therefore, they were not considered for the adjustment. As the sample size was unbalanced between day of illness and DOI 1 was unlikely to have a clinical event, we decided to adjust for DOI rather than to conduct a stratification analysis on this factor. The potentially non-linear effect of CRP levels on the outcomes was investigated using restricted cubic splines for $\log 2$ of CRP levels with three knots at the 10th, 50th, and 90th percentiles [11]. The effect of CRP levels was expressed by odds ratio (OR) for the logistic regression models and hazard ratio (HR) for the Cox regression model. As the effect of CRP levels on the outcome could differ by age, we then performed a sensitivity analysis by subgroups of children $(<15$ years of age) and adults ( $\geq 15$ years of age), using similar statistical method as described above. Because the OFI group included both viral and bacterial infections, this group was further categorized into two subgroups using the level of neutrophil counts: cases with neutrophil counts of $<8 \times 10^{9} / \mathrm{mL}$ (upper limit of normal) were classified as potential viral infection, and the others were classified as potential bacterial infection. CRP levels between dengue and these two subgroups were also explored using similar model. The association between CRP in dengue patients at enrollment and other clinical and biochemical markers (platelet nadir, maximum hematocrit (HCT) change, liver transaminases, creatinine kinase (CK), albumin, white blood cell (WBC) count, percentage of neutrophils and lymphocytes) were explored using scatter plots and Pearson's correlation coefficients. All analyses were performed using the statistical software $\mathrm{R}$ version 3.4.4.

\section{Results}

\section{Patient characteristics}

Samples from 1120 patients with laboratory-confirmed dengue and 400 patients with OFI were tested with CRP. After excluding 9 samples with CRP values above the upper range of the assay, data from 1117 patients with laboratory-confirmed dengue and 394 patients with OFI were included in the final analysis (Fig. 1).

Due to the selection procedure, median age, gender, and DOI at enrollment were very similar between the OFI and dengue group (Table 1). The age range of all patients was 5 to 64 in the OFI group, and 5 to 73 in the dengue group. The number of adults and children enrolled by site can be seen in Additional file 2. Patients with severe dengue were younger $(p<0.001$, MannWhitney $U$ test) than those with other severity grades, and were more likely to be enrolled on day 3 ( $p=0.003$, chi-squared test). In the dengue patients, the percentage of secondary infections was highest in the severe dengue group $33 / 38(86.8 \%)$ and lowest in the uncomplicated dengue group 542/836 (65.2\%) ( $p<0.001$, chi-squared test). Fewer patients with OFI were hospitalized (76/394, $19.3 \%$, versus $421 / 1120,37.6 \%)(p<0.001$, chi-squared test). Within the OFI group, 131/394 (33.2\%) of cases were clinically diagnosed as bacterial infection, while in the dengue group, there were 54/1120 (4.8\%) of cases with clinical diagnosis of bacterial coinfection (see Additional file 3).

\section{Laboratory characteristics}

In patients with dengue infection, median CRP level at enrollment was $30.2 \mathrm{mg} / \mathrm{L}$, similar to the OFI group (median CRP of 31.3) (Table 2). In comparing DOI at enrollment, CRP level was highest in patients enrolled on DOI 2 and lower in patients enrolled on DOI 3 or at follow-up. This trend is similar across dengue severity (Fig. 2). There were 30 patients in the OFI group and 896 patients in the dengue group who had CRP measurements at follow-up (Table 2, Fig. 2). The follow-up days ranged from 10 to 31 days; however, $94 \%$ follow-up samples were within 10-20 days since onset. For the OFI group, while the same trend was seen in patients clinically diagnosed with bacterial infection, CRP level among patients with viral diagnosis was lower for those enrolled 


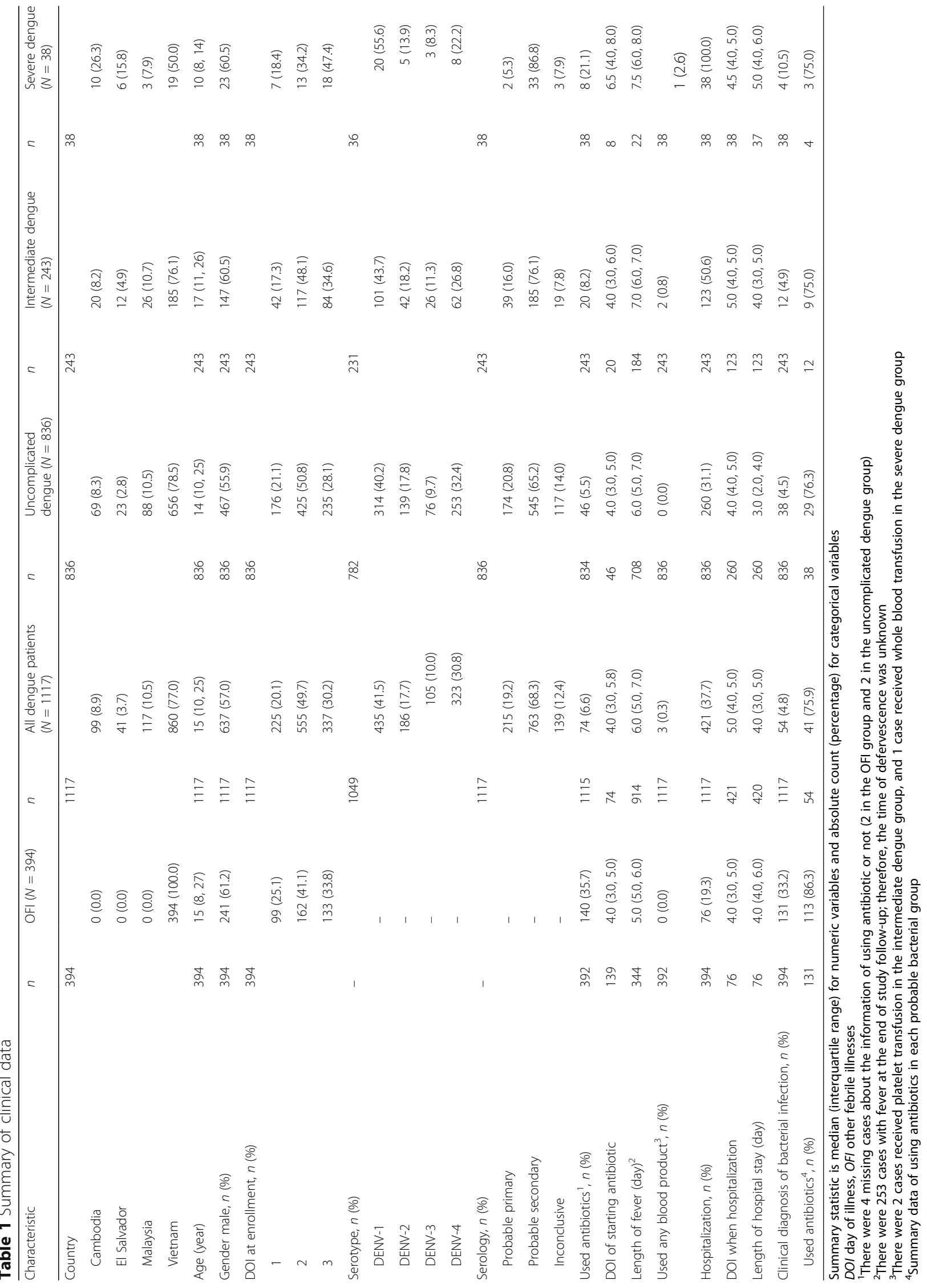




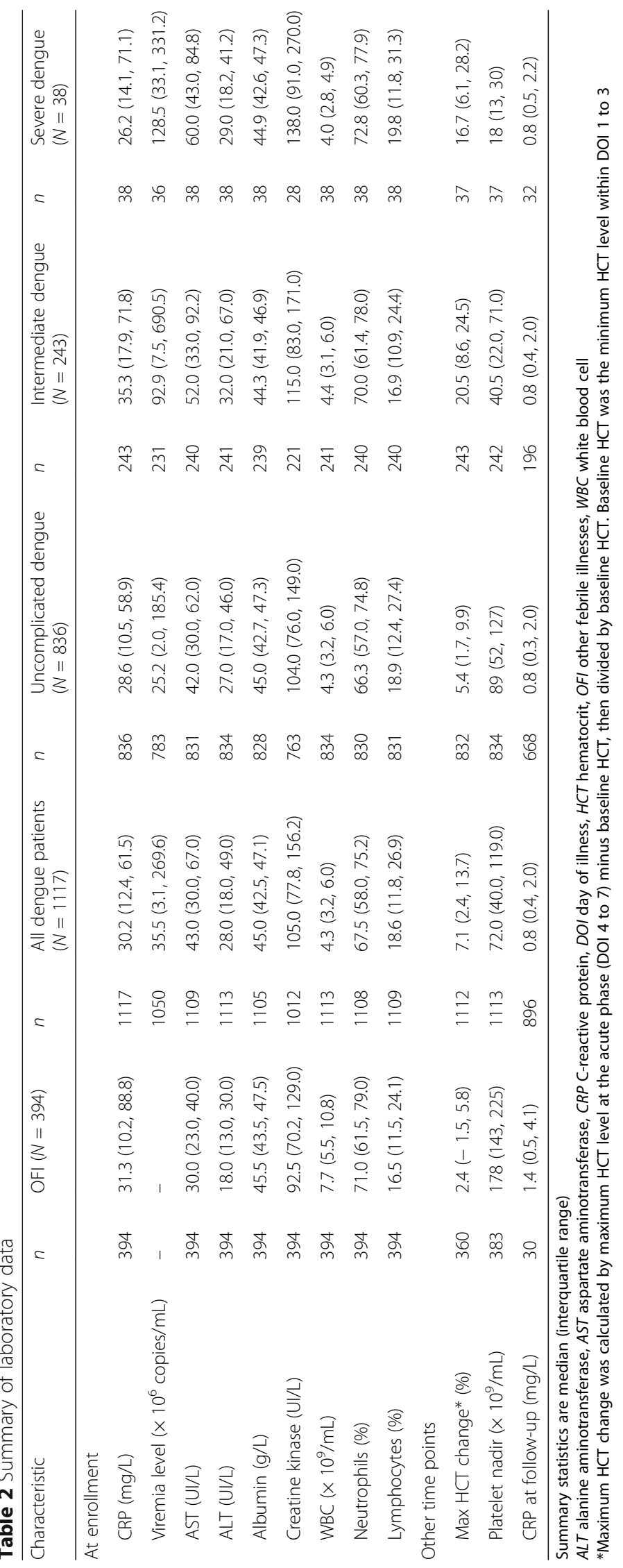




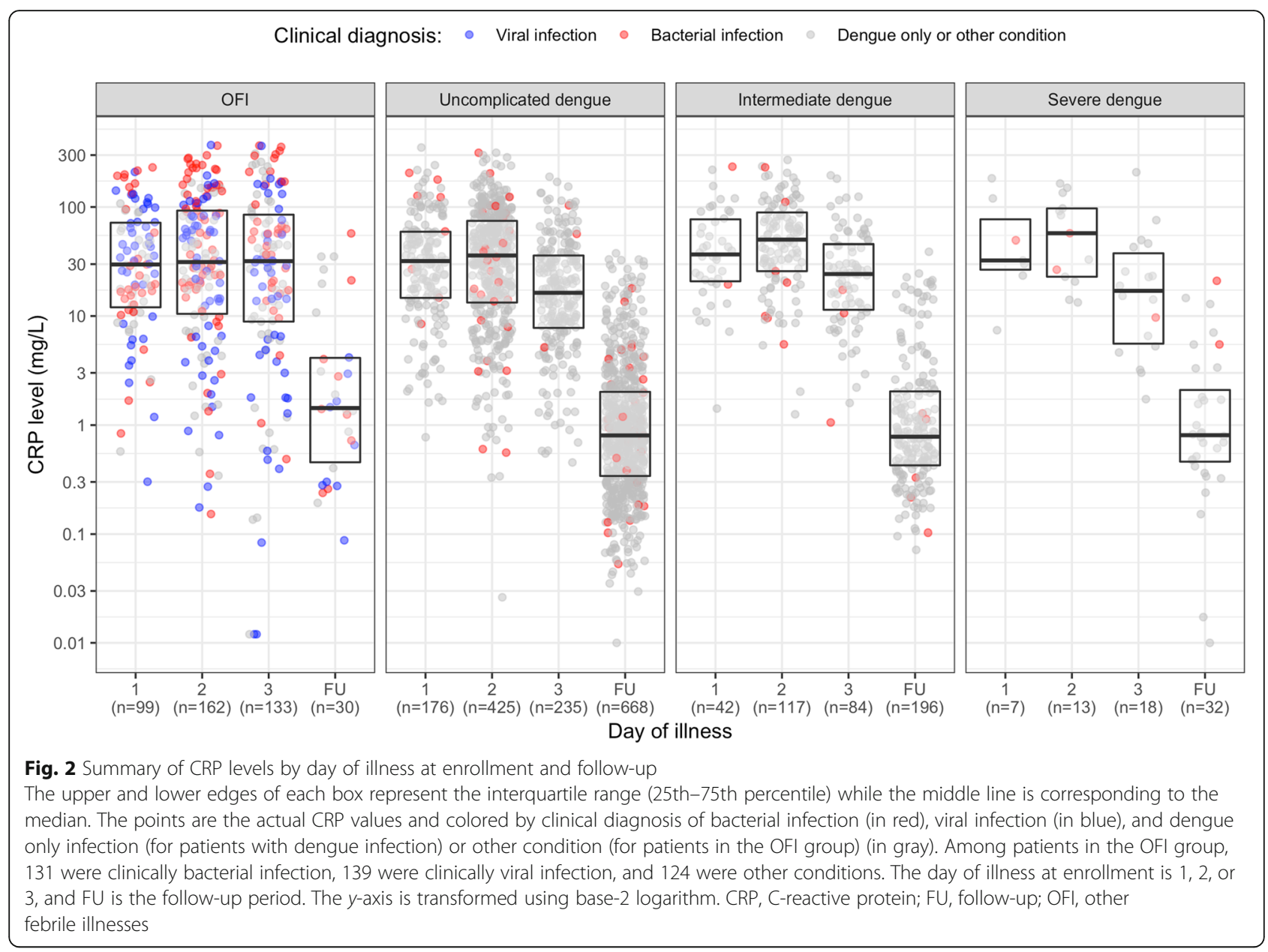

at a later time point. Considering the other laboratory parameters, dengue patients had higher levels of liver transaminases and CK, but lower WBC, and comparable levels of albumin (Table 2). Dengue patients had higher maximum HCT change, but lower PLT nadir. Among patients with dengue, plasma viremia levels were highest in the severe group.

\section{Association of CRP levels and dengue diagnosis}

The OFI group was separated into potential viral or bacterial infections, using the clinical diagnosis and a neutrophil cutoff of $8 \times 10^{9} / \mathrm{mL}$. The boxplots (lower part of Fig. 3) suggest that CRP levels in dengue patients are slightly higher than in patients with probable viral infection $(p=0.003$, Mann-Whitney $U$ test $)$ but lower than in patients with probable bacterial infection ( $p<0.001$, Mann-Whitney $U$ test). These differences resulted in a non-linear relationship between dengue diagnosis and CRP levels, as depicted in the upper plot in Fig. 3. CRP levels of less than $30 \mathrm{mg} / \mathrm{L}$ (approximately the median CRP levels in dengue patients) showed that higher CRP levels associated with higher odds of dengue diagnosis (OR for each two times increase in CRP level was 1.28, 95\% CI 1.181.40). However, in patients with CRP levels $\geq 30 \mathrm{mg} /$ $\mathrm{L}$, higher CRP associated with lower odds of dengue diagnosis (OR for each two times increase in CRP level was $0.64,95 \%$ CI $0.55-0.73$ ). The subgroup analysis of age $<15$ and age $\geq 15$ years showed similar results (see Additional file 4). A sensitivity analysis showed that including the 9 cases with CRP values above the upper range of the assay did not affect the results (ORs [95\% CIs] in patients with CRP levels < 30 and $\geq 30 \mathrm{mg} / \mathrm{L}$ were $1.28[1.18-1.40]$ and 0.63 [0.55-0.72], respectively).

\section{Association of CRP level and clinical outcomes among dengue patients}

The median (IQRs) DOIs of developing the outcomes of intermediate and severe dengue were $5(4 ; 6)$ and $5(5 ; 6)$, and median (IQR) times from the CRP test to these outcomes were $3(2 ; 4)$ and $3(2 ; 4)$ days, respectively. CRP levels at enrollment in patients with severe or intermediate dengue were higher than in patients with uncomplicated dengue, with median levels (interquartile range) of 34.0 (17.4-71.8 mg/L), 


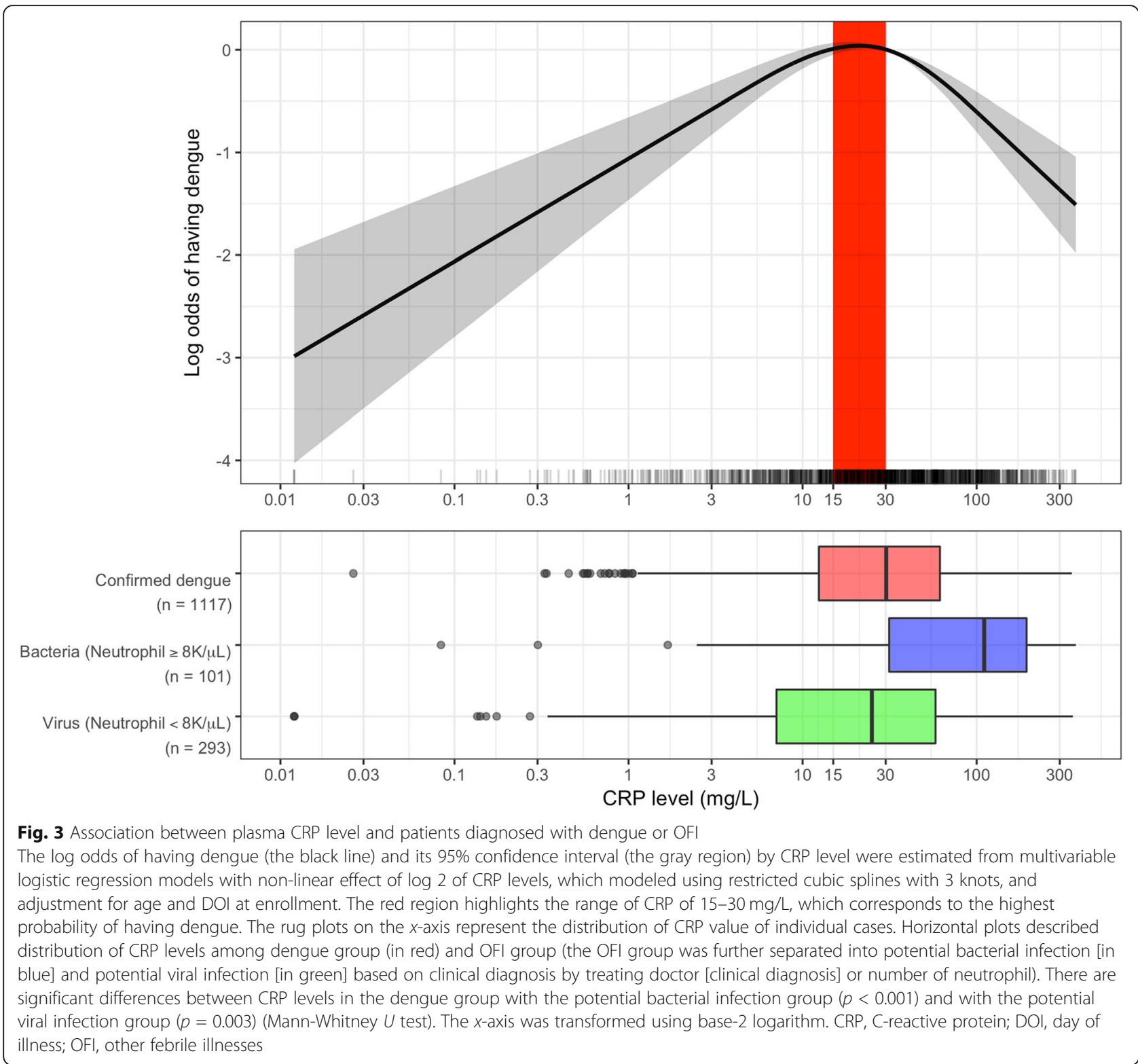

and $28.6 \mathrm{mg} / \mathrm{L}$ (10.5-58.9 mg/L), respectively. Higher CRP levels in the first 3 days of illness were associated with increased odds of severe or intermediate dengue, after correcting for age, DOI at enrollment, plasma viremia levels, and immune status (Table 3). For each twofold increase in CRP level, the OR (95\% CI) of having severe or intermediate dengue was 1.17 (1.07-1.29). Patients with higher CRP level also had higher odds of severe dengue with OR of 1.05 (95\% CI $0.85-1.32$ ), but this did not reach statistical significance. Higher CRP levels in patients with CRP $\geq 30$ $\mathrm{mg} / \mathrm{L}$ were strongly associated with longer fever clearance time (HR 0.84, 95\% CI $0.76-0.93)$ and hospitalization (OR 1.37, 95\% CI 1.14-1.64) (Table 3, Fig. 4). When categorizing CRP levels into 3 groups
$(<15,15-30$, and $\geq 30 \mathrm{mg} / \mathrm{L})$, the 2 latter groups had significantly higher risk of severe or intermediate dengue compared to the lowest CRP level group (ORs [95\% CIs] were 1.66 [1.07-2.56] and 1.59 [1.10-2.32], respectively), after correcting for age, DOI at enrollment, plasma viremia, and immune status.

The subgroup analysis by age, regarding severe or intermediate dengue, showed similar results in children (OR 1.18, 95\% CI 1.04-1.35); however, the magnitude of effect was less in adults (OR 1.10, 95\% CI 0.96-1.27) (see Additional files 5 and 6). The strong association between higher CRP level and longer fever clearance time and hospitalization when CRP level $\geq 30 \mathrm{mg} / \mathrm{L}$ was still apparent in adults (HR of fever clearance was $0.78,95 \%$ CI $0.68-0.89$; OR of hospitalization was $1.59,95 \% \mathrm{CI}$ 
Table 3 Association between CRP level and clinical outcomes among dengue patients

\begin{tabular}{|c|c|c|c|c|c|c|}
\hline Outcome & Crude OR/HR & $95 \% \mathrm{Cl}$ & $p$ value & Adjusted OR/HR & $95 \% \mathrm{Cl}$ & $p$ value \\
\hline Severe or intermediate dengue ${ }^{1}$ & 1.17 & $1.08-1.27$ & $<0.001$ & 1.18 & $1.07-1.30$ & 0.001 \\
\hline Severe dengue $^{1}$ & 1.04 & $0.87-1.25$ & 0.710 & 1.05 & $0.85-1.32$ & 0.638 \\
\hline \multicolumn{7}{|l|}{ Fever clearance time*2 } \\
\hline $\mathrm{CRP}<30 \mathrm{mg} / \mathrm{L}$ & 1.00 & $0.94-1.05$ & 0.866 & 1.03 & $0.97-1.09$ & 0.387 \\
\hline $\mathrm{CRP} \geq 30 \mathrm{mg} / \mathrm{L}$ & 0.89 & $0.81-0.97$ & 0.010 & 0.84 & $0.76-0.92$ & $<0.001$ \\
\hline \multicolumn{7}{|l|}{ Hospitalization ${ }^{2}$} \\
\hline $\mathrm{CRP}<30 \mathrm{mg} / \mathrm{L}$ & 0.93 & $0.83-1.03$ & 0.159 & 0.92 & $0.81-1.04$ & 0.175 \\
\hline $\mathrm{CRP} \geq 30 \mathrm{mg} / \mathrm{L}$ & 1.28 & $1.08-1.51$ & 0.004 & 1.36 & $1.13-1.63$ & 0.001 \\
\hline
\end{tabular}

The estimates (OR and HR) and 95\% Cl were reported for each one log 2 increase of CRP level, i.e., for each 2 times increase of CRP level

$\mathrm{Cl}$ confidence interval, CRP C-reactive protein, DOI day of illness, HR hazard ratio, OR odds ratio

*We use hazard ratio (HR) to report the results of Cox model for fever clearance time outcome. All other outcomes are reported by odds ratio (OR) estimated from logistic regression model. All adjusted estimates are derived from multivariable models adjusted for age, DOI at enrollment, plasma viremia level, and immune status

${ }^{1}$ The models for severe or intermediate dengue and severe dengue were performed with linear effect of log 2 of CRP (the non-linear effect of log 2 of CRP was not statistically significant: $p=0.209$ for severe or intermediate dengue outcome, and $p=0.679$ for severe dengue outcome)

${ }^{2}$ The models for fever clearance time and hospitalization were performed with two separated linear effect of log 2 of CRP (for CRP $<30$ and CRP $\geq 30 \mathrm{mg} / \mathrm{L}$ ). The non-linear effect of log 2 of CRP was statistically significant: $p=0.004$ for fever clearance time outcome, and $p=0.011$ for hospitalization outcome. These models had a quadratic effect with the peak CRP level of approximately $30 \mathrm{mg} / \mathrm{L}$

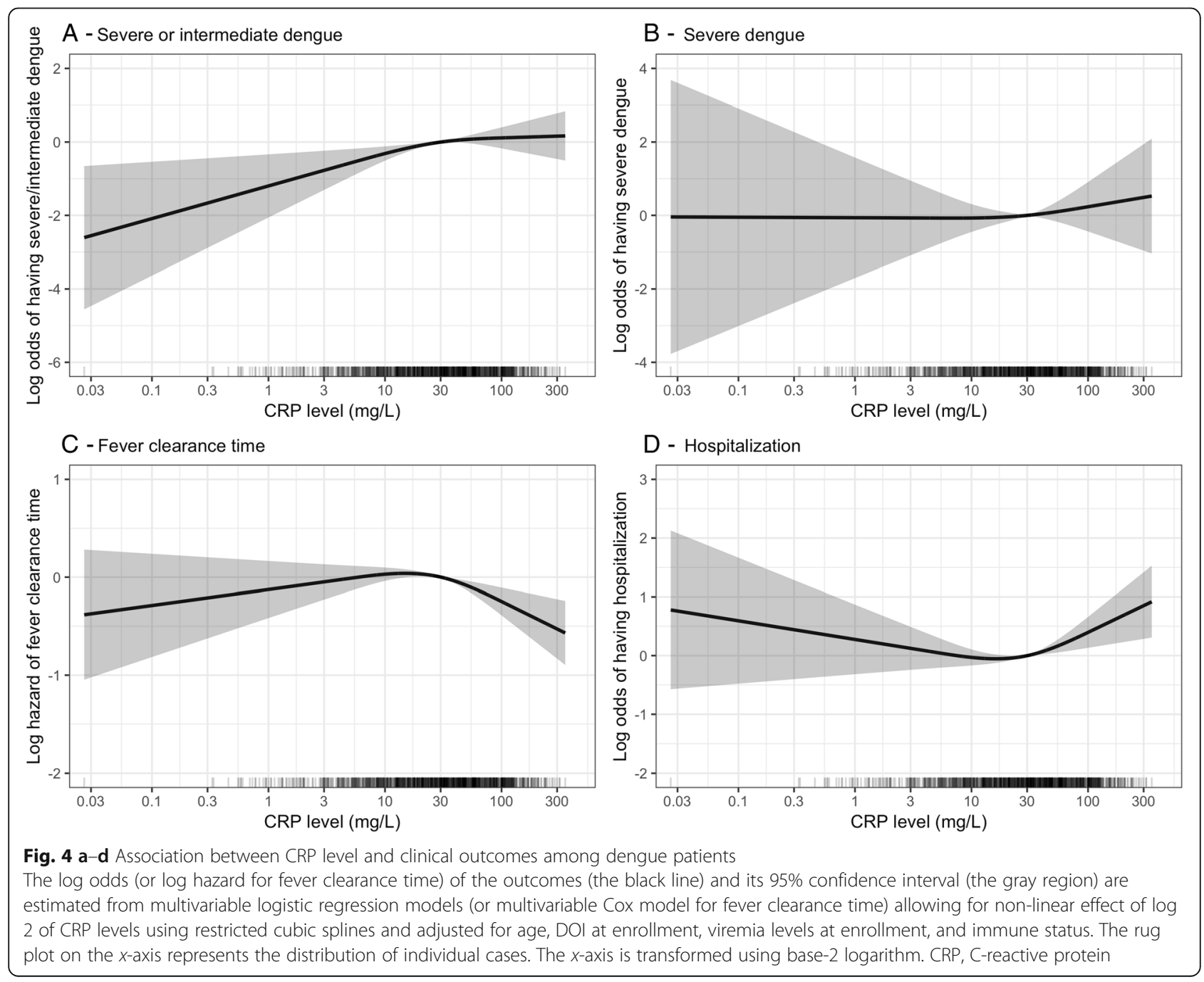


1.25-2.04) but not in children (see Additional files 5, 7, and 8). A sensitivity analysis including 9 cases with CRP values above the upper range of the assay also showed similar results (see Additional file 9).

Overall, antibiotics were used in 140/392 (35.7\%) patients in the OFI group but only in 74/1118 (6.6\%) patients in the dengue group. Among 428 dengue patients with CRP level at enrollment of $>40 \mathrm{mg} / \mathrm{L}$ and without clinically suspected bacterial infection, 417 (97\%) patients did not receive antibiotics (12 from severe dengue group, 107 from intermediate dengue group, and 298 from uncomplicated dengue group).

\section{Association between CRP level and other laboratory tests among dengue patients}

Among dengue patients, we found positive associations between CRP levels and total WBC count and the neutrophil percentage (Pearson's correlation coefficients were 0.25 and 0.3 , respectively), and a negative association between CRP and the lymphocyte percentage (Pearson's correlation coefficient was - 0.36) (Fig. 5). There were no significant associations between CRP level and other laboratory tests including liver transaminases, CK, albumin, maximum HCT change, and PLT nadir (see Additional files 10 and 11).

\section{Discussion}

This large case-control study has shown that increased CRP levels in the first 3 days of illness are associated with worse dengue clinical outcomes, especially in children. These findings provide the most robust evidence of this relationship to date, with a consistent approach across a large and diverse multi-national cohort ensuring maximal generalisability. In identifying an association between higher CRP and a modest increase in the likelihood of progression to intermediate/severe disease, we have demonstrated the potential utility of CRP measured early in the course of infection. This observation could assist patient management at the point of initial presentation, when accurate triage of those most in need of supportive care is imperative. Existing WHO criteria for dengue with warning signs rely heavily on clinical examination findings, with the inclusion of changes in hematocrit and platelet count representing the only laboratory markers of anticipated severity [3]. However, while the importance of these parameters has been emphasized by recent studies [12-14], our findings suggest that the approach to risk stratification could be further refined with the inclusion of baseline CRP.

The prognostic potential of markers of generalized inflammation has previously been suggested, in view of the known immune-enhancing effect inherent to dengue's pathogenicity $[4,15]$. However, recent investigation into the association between CRP and severity of disease has yielded conflicting results [5, 6, 16-18]. Our findings are consistent with those of a smaller study of 191 adults, which reported higher levels of CRP during the febrile phase of illness in severe versus non-severe dengue (defined using 2009 WHO classification) (AUC 0.938 at a CRP threshold of $30.1 \mathrm{mg} / \mathrm{L}$; $100 \%$ sensitivity, $76.3 \%$ specificity) [5]. Other studies have shown a lower range of CRP in dengue infection, but this variability is likely reflective of heterogeneity in laboratory methods used, timing of serum sampling, and classification of clinical outcome. Importantly, each study reporting an absence of association between CRP and severity of disease focused on measures of CRP taken later than 3 days into the course of illness, which may account for their disagreement with our results $[16,17]$.
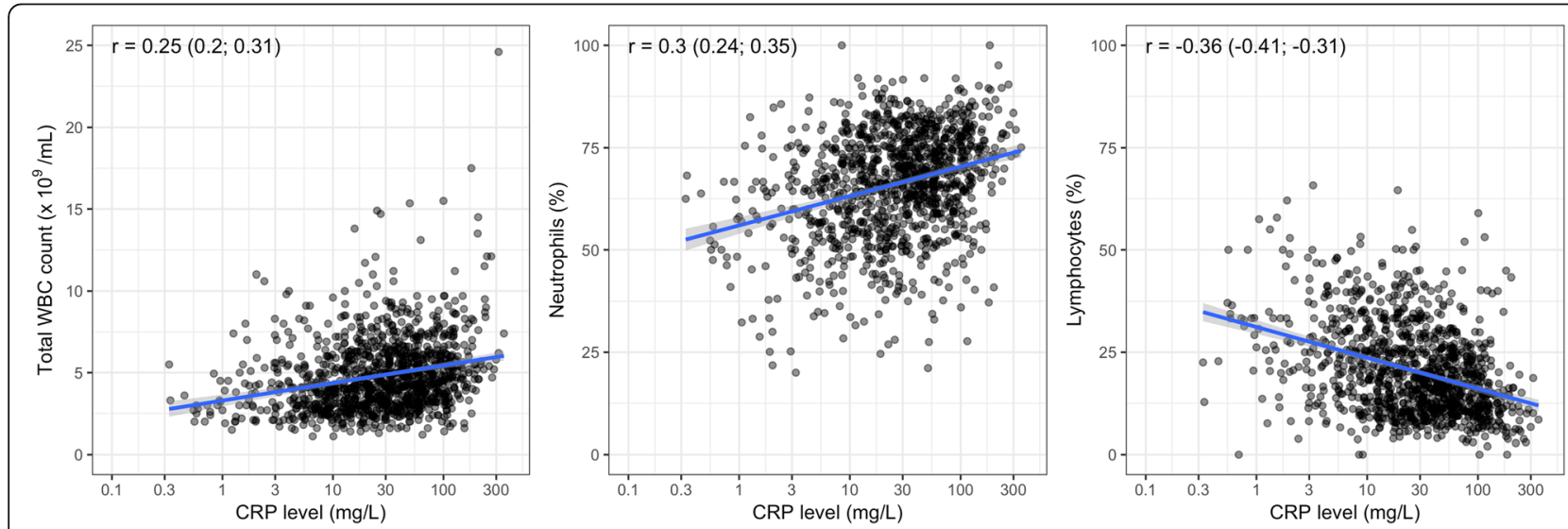

Fig. 5 Association between CRP and total white blood cell count $(n=1115)$, the percentages of neutrophils $(n=1110)$ and lymphocytes $(n=1111)$ Each black point represents for each patient. The blue line is the linear regression line, and the gray region is the 95\% confidence interval. Pearson's correlation coefficient and its $95 \%$ confidence interval are shown in the top left corner of each plot. The $x$-axis is transformed using base-2 logarithm. CRP, C-reactive protein; WBC, white blood cell 
CRP is an acute-phase protein that is rapidly synthesized by hepatocytes in response to inflammatory stimuli. It binds to a number of intrinsic and extrinsic ligands, including many constituents of eukaryotic and prokaryotic pathogens, enabling activation of the classical complement pathway and playing a possible role in alternative-pathway regulation [19]. While the mechanism of association between elevated CRP and dengue remains unclear, immune enhancement is a known feature of dengue pathogenesis, and it may be that severity of disease manifestation is determined by overall magnitude of the immune response. The observed association between higher CRP and increased probability of increased disease severity should serve to inform future clinical trial design, both by enriching study populations and by encouraging exploration of potential antiinflammatory host-directed therapeutics.

Aside from CRP, several other components of the immune response have been identified as potential prognostic biomarkers [4]. Nascimento et al. found associations between markers of dysregulation of the alternative complement pathway and the development of dengue haemorrhagic fever [16], and Juffrie et al. reported levels of the proinflammatory molecules, IL-6 and SPLA2, to be predictive of dengue-associated hypotensive shock [18]. Additional proposed prognostic indicators include a range of proinflammatory cytokines, markers of endothelial activation and microvascular disruption, and acute-phase proteins, but the evidence base to support these associations is limited to small studies with multiple possible confounding sources $[4,17,20]$. The major distinguishing feature that separates CRP from other potential immune biomarkers, however, is its well-established use as a clinical test, with cheap pointof-care (POC) kits readily available across the majority of healthcare settings. It is this ease of access, which makes it a highly attractive option for implementation as a prognostic tool in the resource-limited areas of greatest dengue disease burden.

In addition to addressing the important question of whether CRP can be prognostically useful as a dengue biomarker, our findings add to the existing evidence base for its use in differentiating dengue from nondengue causes of fever $[8,21-30]$. We found the median CRP level of approximately $30 \mathrm{mg} / \mathrm{L}$ among dengue cases within the first 3 days of illness. Other studies found similar levels with observed mean CRP levels of $19.0 \mathrm{mg} / \mathrm{L}$ on the first day of illness in dengue cases [23]. A number of previous studies have demonstrated the role of CRP in differentiating dengue from specifically bacterial [21-26] and malarial infections [25-28], although the most effective CRP thresholds for predicting underlying etiology remain difficult to define. Furthermore, the use of a CRP threshold of $20 \mathrm{mg} / \mathrm{L}$ has been demonstrated in simulation models to be successful and cost-effective in classifying patients into those that would and would not benefit from antibiotics in $80 \%$ of cases, regardless of baseline endemicity levels of different pathogens [24]. In practice, the use of POC CRP assay has been shown to reduce antibiotic prescribing when implementing thresholds of significance of both $20 \mathrm{mg} / \mathrm{L}$ and $40 \mathrm{mg} / \mathrm{L}$ [31]. However, our results highlight the risk of inadvertently increasing inappropriate antibiotic prescribing if dengue is not first considered in patients with CRP $>20 \mathrm{mg} / \mathrm{L}$ during seasons of high incidence. Nevertheless, growing confidence in the diagnostic value of CRP makes POC CRP an increasingly relevant component of antimicrobial stewardship strategy in the present era of ever-growing antimicrobial resistance.

It is important to acknowledge some limitations of our nested case-control study design, with observations relating to the diagnostic value of CRP in dengue infection being particularly susceptible to confounding by selection bias. For example, uncomplicated cases were chosen to match demographic characteristics of the severe/ intermediate group, so the CRP results from these uncomplicated cases cannot be generalized to all uncomplicated dengue cases in the cohort. This effect would be minimized by further exploration in future large prospective cohort studies, including more patients from Latin America. The absence of available data on alternative causes of febrile illness in the study population also makes it difficult to draw firm conclusions about the differentiating power of CRP.

\section{Conclusions}

Despite increasing global efforts to reduce the physical and socioeconomic impact of dengue, reliable biomarkers for predicting disease severity remain scarce. This study provides important insight into the association between higher CRP and risk of disease progression, with results that are generalisable across the range of populations most commonly affected. These findings can be used to influence further research design and health policy on how best to risk-stratify patients at the point of initial assessment.

\section{Supplementary information}

Supplementary information accompanies this paper at https://doi.org/10. 1186/s12916-020-1496-1.

Additional file 1. Clinical endpoint definition.

Additional file 2. Number of adults/children by sites.

Additional file 3. Summary of clinically diagnosis of bacterial infection.

Additional file 4: Figure S1. Association between CRP level and patients diagnosed with dengue or OFI.

Additional file 5. Association between CRP level and clinical outcomes in subgroups of age $<15$ years and age $\geq 15$ years. 
Additional file 6: Figure S2. Association between CRP level and severe or intermediate dengue among dengue patients.

Additional file 7: Figure S3. Association between CRP level and fever clearance time among dengue patients.

Additional file 8: Figure S4. Association between CRP level and hospitalization among dengue patients.

Additional file 9. Association between CRP level and clinical outcomes: sensitivity analysis including 9 cases with CRP values above the upper range of the assay.

Additional file 10: Figure S5. Association between CRP and other laboratory tests result.

Additional file 11. Correlation between CRP and other biomarkers.

\section{Abbreviations}

ALT: Alanine aminotransferase; AST: Aspartate aminotransferase; CK: Creatinine Kinase; CRP: C-reactive protein; DENV: Dengue Virus; DOI: Day of illness; FU: Follow-up; HCT: Haematocrit; HR: Hazard Ratio; HTD: Hospital for Tropical Diseases; IQR: Interquartile range; LOD: Limit of detection; OFI: Other Febrile illness; OR: Odds Ratio; PLT: Platelet; POC: Point of care; RTPCR: Real-time Polymerase chain reaction; WBC: White blood cell

\section{Acknowledgements}

We would like to acknowledge all the patients who took part in this study and the medical and nursing staff who looked helped in their management, at all the participating hospitals in Vietnam, Cambodia, Malaysia, and El Salvador.

\section{Authors' contributions}

SY, BW, PO, CH, and TJ designed the study and edited the manuscript. NLV and PKL performed the statistical analysis and wrote the results. HTLD performed the laboratory procedures and assisted in writing the manuscript. DTHT, NWC, NVK, NC, LL, and EP recruited and managed the patients at each site. $N J$ analyzed the data and assisted in writing the manuscript. CPS and KDR provided intellectual input to the study and the manuscript. All authors read and approved the final manuscript.

\section{Funding}

This work was supported by the World Health Organization, UNICEF/UNDP/ World Bank/WHO Special Programme for Research and Training in Tropical Diseases, and the European Union's Seventh Framework Programme for research, technological development and demonstration (grant FP7-281803 IDAMS; http://www.idams.eu/; publication reference number IDAMS: 53) The funders had no role in the study design, data collection and analysis, or preparation of the manuscript.

\section{Availability of data and materials}

Dataset available from the OUCRU repository—on request to the corresponding author (S Yacoub).

\section{Ethics approval and consent to participate}

Ethical Committee approval was obtained from Oxford Tropical Research Ethics Committee (OxTREC) in the UK, and from all the local IRBs at each site for the original observational study. Further minimal risk applications were obtained for use of stored samples from OxTREC, and all other participating hospitals from which residual samples were selected. All patients signed an informed consent form in the original study, which included permission for future use of stored blood samples.

\section{Consent for publication}

Not applicable.

\section{Competing interests}

SY receives consulting fees from Janssen pharmaceuticals for work on dengue antiviral development. All other authors declared that they have no competing interests. All authors have submitted the ICMJE Form for Disclosure of Potential Conflicts of Interest.

$\mathrm{CH}$ is staff of the World Health Organization. The authors alone are responsible for the views expressed in this article, and they do not necessarily represent the views, decisions, or policies of the institutions with which they are affiliated.

\section{Author details}

${ }^{1}$ Oxford University Clinical Research Unit, Wellcome Trust Asia Programme, Ho Chi Minh City, Vietnam. ${ }^{2}$ University of Medicine and Pharmacy at Ho Chi Minh City, Ho Chi Minh City, Vietnam. ${ }^{3}$ Hospital of Tropical Diseases, Ho Chi Minh City, Vietnam. ${ }^{4}$ National Hospital for Tropical Diseases (NHTD), Hanoi, Vietnam. ${ }^{5}$ Angkor Hospital for Children, Siem Reap, Cambodia. ${ }^{6}$ University of Malaya Medical Centre, Kuala Lumpur, Malaysia. ${ }^{7}$ Hospital Nacional de Niños Benjamin Bloom, San Salvador, El Salvador. ${ }^{8}$ University of Cambridge, Cambridge, UK. 'Institute of Vector-Borne Disease, Monash University, Melbourne, Australia. ${ }^{10}$ Section of Clinical Tropical Medicine, Department of Infectious Diseases, Heidelberg University Hospital, Heidelberg, Germany. ${ }^{11}$ UNICEF/UNDP/World Bank/WHO Special Programme for Research and Training in Tropical Diseases, World Health Organization, Geneva, Switzerland. ${ }^{12}$ Centre for Tropical Medicine and Global Health, Nuffield Department of Medicine, University of Oxford, Oxford, UK.

Received: 8 August 2019 Accepted: 13 January 2020

Published online: 17 February 2020

References

1. Simmons CP, Farrar JJ, Nguyen V, Wills B. Dengue. N Engl J Med. 2012; 366(15):1423-32.

2. Bhatt $S$, Gething PW, Brady OJ, et al. The global distribution and burden of dengue. Nature. 2013;496(7446):504-7.

3. World Health Organization. Dengue: guidelines for treatment, prevention and control. New Edition. Geneva: World Health Organization; 2009.

4. Yacoub S, Wills B. Predicting outcome from dengue. BMC Med. 2014;12:147.

5. Chen CC, Lee IK, Liu JW, Huang SY, Wang L. Utility of C-reactive protein levels for early prediction of dengue severity in adults. Biomed Res Int. 2015; 2015:936062.

6. Eppy S, Nainggolan L, Rumende CM. The differences between interleukin- 6 and c-reactive protein levels among adult patients of dengue infection with and without plasma leakage. Acta Med Indones. 2016;48(1):3-9.

7. Durán A, González A, Delgado L, Mosquera J, Valero N. Serum level of Creactive protein is not a parameter to determine the difference between viral and atypical bacterial infections. J Med Virol. 2016;88(2):351-5.

8. Feitosa RN, Vallinoto AC, Vasconcelos PF, et al. Gene polymorphisms and serum levels of pro- and anti-inflammatory markers in dengue viral infections. Viral Immunol. 2016:29(7):379-88.

9. Jaenisch T, Tam DT, Kieu NT, et al. Clinical evaluation of dengue and identification of risk factors for severe disease: protocol for a multicentre study in 8 countries. BMC Infect Dis. 2016;16:120.

10. Tomashek KM, Wills B, See Lum LC, et al. Development of standard clinical endpoints for use in dengue interventional trials. PLoS Negl Trop Dis. 2018; 12(10):e0006497.

11. Durrleman S, Simon R. Flexible regression models with cubic splines. Stat Med. 1989 May;8(5):551-61.

12. Ahmad MH, Ibrahim MI, Mohamed $Z$, et al. The sensitivity, specificity and accuracy of warning signs in predicting severe dengue, the severe dengue prevalence and its associated factors. Int J Environ Res Public Health. 2018; 15(9).

13. Phakhounthong $K$, Chaovalit $P$, Jittamala $P$, et al. Predicting the severity of dengue fever in children on admission based on clinical features and laboratory indicators: application of classification tree analysis. BMC Pediatr. 2018;18(1):109.

14. Tamibmaniam J, Hussin N, Cheah WK, Ng KS, Muninathan P. Proposal of a clinical decision tree algorithm using factors associated with severe dengue infection. PLoS One. 2016;11(8):e0161696.

15. Diamond MS, Pierson TC. Molecular insight into dengue virus pathogenesis and its implications for disease control. Cell. 2015;162(3):488-92.

16. Nascimento EJ, Silva AM, Cordeiro MT, et al. Alternative complement pathway deregulation is correlated with dengue severity. PLoS One. 2009; 4(8):e6782.

17. Conroy AL, Gélvez M, Hawkes M, et al. Host biomarkers are associated with progression to dengue haemorrhagic fever: a nested case-control study. Int J Infect Dis. 2015:40:45-53. 
18. Juffrie $M$, Meer $G M$, Hack $C E$, et al. Inflammatory mediators in dengue virus infection in children: interleukin-6 and its relation to C-reactive protein and secretory phospholipase A2. Am J Trop Med Hyg. 2001;65(1):70-5.

19. Pepys MB, Hirschfield GM. C-reactive protein: a critical update. J Clin Invest. 2003;111(12):1805-12.

20. Mairuhu AT, Peri G, Setiati TE, et al. Elevated plasma levels of the long pentraxin, pentraxin 3, in severe dengue virus infections. J Med Virol. 2005; 76(4):547-52.

21. Prodjosoewojo S, Riswari SF, Djauhari H, et al. A novel diagnostic algorithm equipped on an automated hematology analyzer to differentiate between common causes of febrile illness in Southeast Asia. PLoS Negl Trop Dis. 2019;13(3):e0007183.

22. Wangrangsimakul T, Althaus T, Mukaka M, et al. Causes of acute undifferentiated fever and the utility of biomarkers in Chiangrai, northern Thailand. PLoS Negl Trop Dis. 2018;12(5):e0006477.

23. Chen CH, Huang YC, Kuo KC, Li CC. Clinical features and dynamic ordinary laboratory tests differentiating dengue fever from other febrile illnesses in children. J Microbiol Immunol Infect. 2018;51(5):614-20.

24. Lubell Y, Althaus T, Blacksell SD, et al. Modelling the impact and costeffectiveness of biomarker tests as compared with pathogen-specific diagnostics in the management of undifferentiated fever in remote tropical settings. PLoS One. 2016;11(3):e0152420.

25. Chang K, Lee NY, Ko WC, et al. Identification of factors for physicians to facilitate early differential diagnosis of scrub typhus, murine typhus, and Q fever from dengue fever in Taiwan. J Microbiol Immunol Infect. 2017;50(1): 104-11.

26. Cooper EC, Ratnam I, Mohebbi M, Leder K. Laboratory features of common causes of fever in returned travelers. J Travel Med. 2014;21(4):235-9.

27. Kutsuna S, Hayakawa K, Kato Y, et al. The usefulness of serum C-reactive protein and total bilirubin levels for distinguishing between dengue fever and malaria in returned travelers. Am J Trop Med Hyg. 2014;90(3):444-8.

28. Kutsuna S, Hayakawa K, Kato Y, et al. Comparison of clinical characteristics and laboratory findings of malaria, dengue, and enteric fever in returning travelers: 8-year experience at a referral center in Tokyo, Japan. Kansenshogaku Zasshi. 2015;21(4):272-6. https://doi.org/10.1016/j.jiac.2014. 12.004 .

29. Epelboin $L$, Boullé $C$, Ouar-Epelboin $S$, et al. Discriminating malaria from dengue fever in endemic areas: clinical and biological criteria, prognostic score and utility of the C-reactive protein: a retrospective matched-pair study in French Guiana. PLoS Negl Trop Dis. 2013;7(9):e2420.

30. Ho TS, Wang SM, Lin YS, Liu CC. Clinical and laboratory predictive markers for acute dengue infection. J Biomed Sci. 2013;20:75.

31. Althaus T, Greer RC, Swe MMM, et al. Effect of point-of-care C-reactive protein testing on antibiotic prescription in febrile patients attending primary care in Thailand and Myanmar: an open-label, randomised, controlled trial. Lancet Glob Health. 2019;7(1):e119-e31.

\section{Publisher's Note}

Springer Nature remains neutral with regard to jurisdictional claims in published maps and institutional affiliations.

\section{Ready to submit your research? Choose BMC and benefit from:}

- fast, convenient online submission

- thorough peer review by experienced researchers in your field

- rapid publication on acceptance

- support for research data, including large and complex data types

- gold Open Access which fosters wider collaboration and increased citations

- maximum visibility for your research: over $100 \mathrm{M}$ website views per year

At $\mathrm{BMC}$, research is always in progress.

Learn more biomedcentral.com/submissions 